Pure and Applied Mathematics Quarterly

Volume 6, Number 4

(Special Issue: In honor of

Joseph J. Kohn, Part 2 of 2$)$

1027-1033, 2010

\title{
Formal Meromorphic Functions on Manifolds of Finite Type
}

\author{
Robert Juhlin, Bernhard Lamel and Francine Meylan \\ Dedicated to Professor J.J.Kohn on the occasion of his 75th birthday
}

\begin{abstract}
It is shown that a real-valued formal meromorphic function on a formal generic submanifold of finite Kohn-Bloom-Graham type is necessarily constant.
\end{abstract}

\section{INTRODUCTION}

It is easy to see (and known, see [1]) that if $M \subset \mathbb{C}^{N}$ is a connected generic real-analytic CR manifold which is of finite type in the sense of Kohn [5] and Bloom-Graham [4] at some point $p \in M$, then any meromorphic map $H: U \rightarrow \mathbb{C}^{m}$ defined on a connected neighbourhood of $M$ which satisfies $H(M) \subset E$, where $E \subset \mathbb{C}^{m}$ is a totally real real-analytic submanifold, is necessarily constant.

Let us give a short proof of this fact. First, we recall the definition of the Segre sets $S_{p}^{j}$. These are defined inductively. First, we define the Segre variety $S_{p}=S_{p}^{1}$

Received December 11, 2007

The authors were supported by the Austrian Science Fund FWF, project P19667; the third author was partially supported by Swiss NSF grant 2100-063464.00/2. This paper was written during the third author's visit to Vienna; she wishes to express her gratitude for the hospitality of the faculty of mathematics at the University of Vienna. 
for $p \in M$. Let $\rho(Z, \bar{Z})=\left(\rho_{1}(Z, \bar{Z}), \ldots, \rho_{d}(Z, \bar{Z})\right)$ be a (vector-valued) defining function for $M$ defined in a neighbourhood $U \times \bar{U}$ of $(p, \bar{p})$, i.e.

$$
M \cap U=\{Z \in U: \rho(Z, \bar{Z})=0\}, \quad d \rho_{1} \wedge \cdots d \rho_{d} \neq 0 \text { on } \mathrm{U}, \quad \rho(Z, \bar{Z})=\bar{\rho}(\bar{Z}, Z) .
$$

Then if $S_{q}^{1}$ is defined by

$$
S_{q}^{1}=\{Z \in U: \rho(Z, \bar{q})=0\}, \quad q \in U,
$$

the $j$-th Segre set $S_{p}^{j}, j \in \mathbb{N}$, is defined inductively by

$$
S_{p}^{j}=\bigcup_{q \in S_{p}^{j-1}} S_{q}^{1} .
$$

We are using the following Theorem, which characterizes finite type in terms of properties of the Segre sets:

Theorem 1 (Baouendi, Ebenfelt and Rothschild [1]). Let $M \subset \mathbb{C}^{N}$ be a generic real-analytic $C R$ manifold. Then $M$ is of finite type at $p \in M$ if and only if there exists an open set $V \subset \mathbb{C}^{N}$ with $V \subset S_{p}^{d+1}$.

Now assume that $H: U \rightarrow \mathbb{C}^{m}$ is a meromorphic map which satisfies $H(M) \subset$ $E$, where $E$ is totally real. First note that since $M$ is of finite type at some point $p$, it is of finite type on the complement of a proper real-analytic subvariety $F \subset M$. So there exists a point $p \in M$ with the property that $M$ is of finite type at $p$ and $H$ is holomorphic in some neighbourhood of $p$ (because $M$ is generic, it is a set of uniqueness for holomorphic functions). We shall prove that in this situation, $H$ is constant on an open set in $\mathbb{C}^{N}$, and thus constant.

We can find coordinates $\eta$ in $\mathbb{C}^{m}$ such that near $H(p), E$ is given by an equation of the form $\eta=\varphi(\bar{\eta})$. Thus, $H(Z)=\varphi(\bar{H}(\bar{Z}))$, whenever $Z \in M$, and from this we have that $H(Z)=\varphi(\bar{H}(\zeta))$ whenever $Z \in S_{\zeta}$ (restricting to a suitable neighbourhood $U$ of $p$ ). Thus, $H(Z)=\varphi(\bar{H}(p))$ for $Z \in S_{p}$; since $p \in S_{p}$, $H(Z)=H(p)$ for $Z \in S_{p}$. Now we consider $Z \in S_{p}^{2}$. For each such $Z$, there is $\zeta \in S_{p}^{1}$ with $Z \in S_{\zeta}^{1}$. Our equation tells us that $H(Z)=\varphi(\bar{H}(\zeta))=\varphi(\bar{H}(p))$, and again, since $p \in S_{p}^{2}, H(Z)=H(p)$ for $Z \in S_{p}^{2}$.

Continuing the iteration process like this, we see that $H(Z)=H(p)$ for $Z \in S_{p}^{j}$ for $j \in \mathbb{N}$. Since $S_{p}^{d+1}$ contains an open subset of $\mathbb{C}^{N}$ by Theorem 1 , the identity principle implies that $H(Z)=H(p)$ on $U$. This proves the constancy of such an $H$. 
Our main point in this paper is the extension of this result to the formal category. Here we cannot "move to a good point". In this setting, a formal meromorphic map is given by $H=\frac{N}{D}$, where $D$ is a (nonvanishing) formal power series and $N:\left(\mathbb{C}^{N}, 0\right) \rightarrow\left(\mathbb{C}^{m}, 0\right)$ is a formal holomorphic map. Note that if $E \subset \mathbb{C}^{m}$ is a formal totally real manifold, then in suitable coordinates $\eta \in \mathbb{C}^{m}$, $E$ is given by $\operatorname{Im} \eta=0$. We say that $H=N / D$ maps $M$ into $E$ if for any formal map $\gamma(t)=\left(\gamma_{1}(t), \gamma_{2}(t)\right)$ satisfying $\rho\left(\gamma_{1}(t), \gamma_{2}(t)\right)=0$ for every defining function $\rho$ of $M$ we have

$$
N_{j}\left(\gamma_{1}(t)\right) \bar{D}\left(\gamma_{2}(t)\right)-\bar{N}_{j}\left(\gamma_{2}(t)\right) D\left(\gamma_{1}(t)\right)=0
$$

for every $j=1, \ldots, m$. We shall freely use the terminology of formal real submanifolds as explained in e.g. [2]. We show the following:

Theorem 2. Let $M \subset \mathbb{C}^{N}$ be a formal generic manifold of finite type, $H:\left(\mathbb{C}^{N}, 0\right) \rightarrow\left(\mathbb{C}^{m}, 0\right)$ a formal meromorphic map which satisfies $H(M) \subset E$, where $E$ is a formal totally real manifold. Then $H$ is formal holomorphic, and thus, constant.

We note that the finite type assumption is necessary. Indeed, every manifold of the form $M=\tilde{M} \times E$ where $\tilde{M}$ is some CR manifold and $E$ is totally real has nonconstant $\mathrm{CR}$ maps onto a totally real manifold (the projection onto its second coordinate). On the other hand, here is another example, due to J. Lebl:

Example 1. Let $M \subset \mathbb{C}^{3}$ be given by

$$
w_{1}=\overline{w_{1}} e^{i p|z|^{2}}, \quad w_{2}=\overline{w_{2}} e^{i q|z|^{2}},
$$

for some integers $p$ and $q$. Then the function

$$
H\left(z, w_{1}, w_{2}\right)=\frac{w_{1}^{q}}{w_{2}^{p}}
$$

maps $M$ into $\mathbb{R}$ and is not the restriction of a holomorphic function. Also note that this function is not even continuous on $M$. Our results imply that no nonconstant holomorphic choice of projection onto $\mathbb{R}$ can be made.

\section{Reflection Identities and Consequences}

We shall first show that we can simplify our situation somewhat by choosing "normal" coordinates. Recall that normal coordinates for a formal generic 
submanifold $(M, 0) \subset\left(\mathbb{C}^{N}, 0\right)$ means a choice of coordinates $(z, w) \in \mathbb{C}^{n} \times \mathbb{C}^{d}(d$ being the real codimension of $(M, 0))$ together with formal functions $Q_{j}(z, \chi, \tau) \in$ $\mathbb{C}[[z, \chi, \tau]], j=1, \ldots, d$, satisfying

$$
Q_{j}(z, 0, \tau)=Q_{j}(0, \chi, \tau)=\tau_{j}, \quad j=1, \ldots, d,
$$

such that $w_{j}-Q_{j}(z, \chi, \tau)$ generate the manifold ideal associated to $(M, 0)$ in $\mathbb{C}[[z, w, \chi, \tau]]$. We will write $Q=\left(Q_{1}, \ldots, Q_{d}\right)$, and abbreviate the generating set with $w-Q(z, \chi, \tau)$.

We will show that in normal coordinates, a formal meromorphic function $H$ which maps $(M, 0)$ into $(\mathbb{R}, 0)$ actually only depends on the transverse variables $w$. To do this, we first give a reflection identity which we will use.

Proposition 1. If $(M, 0) \subset\left(\mathbb{C}^{N}, 0\right)$ is a formal generic submanifold, and $(z, w)$ are normal coordinates for $(M, 0)$ with corresponding generators $w-Q(z, \chi, \tau)$. If $H=\frac{N}{D}:(M, 0) \rightarrow(\mathbb{R}, 0)$ is formal meromorphic, and $N$ and $D$ do not have any common factors, then there exists a formal holomorphic function $a\left(z, \chi, z^{1}, w\right)$, with $a(0,0,0,0)=1$, such that

$$
\begin{aligned}
& N\left(z, Q\left(z, \chi, \bar{Q}\left(\chi, z^{1}, w\right)\right)\right)=a\left(z, \chi, z^{1}, w\right) N\left(z^{1}, w\right), \\
& D\left(z, Q\left(z, \chi, \bar{Q}\left(\chi, z^{1}, w\right)\right)\right)=a\left(z, \chi, z^{1}, w\right) D\left(z^{1}, w\right) .
\end{aligned}
$$

Proof. The conclusion is clear if $N$ is identically zero, so we assume that this is not the case. By definition, we have

$$
\bar{D}(\chi, \tau) N(z, Q(z, \chi, \tau))=\bar{N}(\chi, \tau) D(z, Q(z, \chi, \tau)) .
$$

Taking the complex conjugate of the series and replacing $\chi$ by $z^{1}, \tau$ by $w$, and $z$ by $\chi$ in this equation, we also have that

$$
D\left(z^{1}, w\right) \bar{N}\left(\chi, \bar{Q}\left(\chi, z^{1}, w\right)\right)=N\left(z^{1}, w\right) \bar{D}\left(\chi, \bar{Q}\left(\chi, z^{1}, w\right)\right) .
$$

We now substitute $\tau=\bar{Q}\left(\chi, z^{1}, w\right)$ into (2) to obtain

$$
\begin{aligned}
& \bar{D}\left(\chi, \bar{Q}\left(\chi, z^{1}, w\right)\right) N\left(z, Q\left(z, \chi, \bar{Q}\left(\chi, z^{1}, w\right)\right)\right) \\
& =\bar{N}\left(\chi, \bar{Q}\left(\chi, z^{1}, w\right)\right) D\left(z, Q\left(z, \chi, \bar{Q}\left(\chi, z^{1}, w\right)\right)\right) .
\end{aligned}
$$

We now multiply the left (and right, respectively) hand sides of (3) and (4) with each other, and after cancelling the (nonvanishing) common factor $\bar{N}\left(\chi, \bar{Q}\left(\chi, z^{1}, w\right)\right) \bar{D}\left(\chi, \bar{Q}\left(\chi, z^{1}, w\right)\right)$ we obtain

$$
D\left(z^{1}, w\right) N\left(z, Q\left(z, \chi, \bar{Q}\left(\chi, z^{1}, w\right)\right)\right)=D\left(z, Q\left(z, \chi, \bar{Q}\left(\chi, z^{1}, w\right)\right)\right) N\left(z^{1}, w\right) .
$$


Now, using the fact that $N$ and $D$ do not have any common factors, unique factorization in the ring $\mathbb{C}\left[\left[z, \chi, z^{1}, w\right]\right]$ implies that there exists a unit $a\left(z, \chi, z^{1}, w\right)$ such that (1) holds. By evaluating (1) at $z=z^{1}$, and using the reality property $Q(z, \chi, \bar{Q}(\chi, z, w))=w$, we have that $a(z, \chi, z, w)=1$, so in particular, $a(0,0,0,0)=1$.

Lemma 2. Let $(M, 0) \subset\left(\mathbb{C}^{N}, 0\right)$ be a formal generic submanifold. Assume that $H(Z)=\frac{N(Z)}{D(Z)}$ is a formal meromorphic map sending $(M, 0)$ into $(\mathbb{R}, 0)$. Then for any choice of normal coordinates $(z, w)$ for $(M, 0)$, we have that $H(z, w)=$ $H(0, w)$; i.e., there exist formal functions $\tilde{N}(w)$ and $\tilde{D}(w)$ such that $H(z, w)=$ $\frac{\tilde{N}(w)}{\tilde{D}(w)}$.

Proof. We use Proposition 1. Setting $\chi=z^{1}=0$, we see that

$$
N(z, w)=a(z, 0,0, w) N(0, w), \quad D(z, w)=a(z, 0,0, w) D(0, w) .
$$

The Lemma follows.

\section{Prolongation of the reflection along Segre maps and proof of THEOREM 2}

We will denote by

$$
v^{1}\left(z, \chi, z^{1} ; w\right)=Q\left(z, \chi, \bar{Q}\left(\chi, z^{1}, w\right)\right)
$$

in the usual Segre-map terminology, $v^{1}\left(z, \chi, z^{1} ; 0\right)$ is the transversal component of the second Segre map of $(M, 0)$. We define $S^{(0)}=z$, and for $j \geq 1$

$$
S^{(j)}=\left(z, \chi, z^{1}, \chi^{1}, \ldots, z^{j}\right),
$$

and write $S_{k}^{(j)}=\left(z^{k}, \chi^{k}, \ldots, z^{j}\right)$ for $k \leq j$. With that notation and our simplification from Lemma 2, our reflection identity (1) now reads

$$
\begin{aligned}
& N\left(v^{1}\left(S^{(1)} ; w\right)\right)=a\left(S^{(1)}, w\right) N(w), \\
& D\left(v^{1}\left(S^{(1)} ; w\right)\right)=a\left(S^{(1)}, w\right) D(w) .
\end{aligned}
$$

For $j \geq 2$, we define inductively

$$
v^{j}\left(S^{(j)} ; w\right)=v^{1}\left(z, \chi, z^{1} ; v^{j-1}\left(S_{1}^{(j)} ; w\right)\right) .
$$

We can now state the finite type criterion of Baouendi, Ebenfelt and Rothschild [3], for later reference, as follows: 
Theorem 3. If $(M, 0)$ is of finite type in the sense of Kohn-Bloom-Graham, then there exists a $j \geq 1$ such that

$$
S^{(j)} \mapsto v^{j}\left(S^{(j)} ; 0\right), \quad\left(\mathbb{C}^{(2 j-1) n}, 0\right) \rightarrow\left(\mathbb{C}^{d}, 0\right),
$$

is of generic full rank $d$.

Thus, if we for $j \geq 2$ replace $w$ by $v^{j-1}\left(S_{1}^{(j)} ; w\right)$ in $(6)$, we obtain

$$
\begin{aligned}
N\left(v^{j}\left(S^{(j)} ; w\right)\right) & =N\left(v^{1}\left(S^{(1)} ; v^{j-1}\left(S_{1}^{(j)} ; w\right)\right)\right) \\
& =a\left(S^{(1)} ; v^{j-1}\left(S_{1}^{(j)} ; w\right)\right) N\left(v^{j-1}\left(S_{1}^{(j)} ; w\right)\right) .
\end{aligned}
$$

Applying induction, we see that the following holds:

Lemma 3. For every $j \geq 1$, there exists a unit $a_{j}\left(S^{(j)}, w\right)$ such that

$$
N\left(v^{j}\left(S^{(j)} ; w\right)\right)=a_{j}\left(S^{(j)}, w\right) N(w), \quad D\left(v^{j}\left(S^{(j)} ; w\right)\right)=a_{j}\left(S^{(j)}, w\right) D(w) .
$$

We can now prove Theorem 2: By Theorem 3, there exists a $j$ such that $v^{j}\left(S^{(j)} ; 0\right)$ is of generic full rank. Assuming that $D(0)=0$, we see that $D\left(v^{j}\left(S^{(j)} ; 0\right)\right)=0$. Since $v^{j}$ is of generic full rank, this implies that $D(w)=0$; this contradiction shows that $D(0) \neq 0$. Hence, we can assume that $H(w)=N(w)$ is holomorphic, and without loss of generality, $N(0)=0$. Now the same argument as before shows that $N(w)=0$, and so, $H$ is constant.

Remark 1. More generally, if we do not assume that $(M, 0)$ is of finite type, then we can define the formal variety

$$
V_{j}=\overline{\operatorname{image}\left(v^{j}\left(S^{(j)} ; 0\right)\right)} \cong\left\{f \in \mathbb{C}[[w]]: f \circ v^{j}\left(S^{(j)} ; 0\right)=0\right\},
$$

and $V=\cup_{j} V_{j}$ (which is again a formal variety). The same arguments as above show that $D$, as well as $N$, are constant on $V$. This corresponds to the statement that a real-valued $\mathrm{CR}$ meromorphic function is constant along the CR-orbits of $M$.

\section{REFERENCES}

[1] M. S. Baouendi, P. Ebenfelt, and L. P. Rothschild. Algebraicity of holomorphic mappings between real algebraic sets in $\mathbb{C}^{n}$. Acta Math., 177(2):225-273, 1996.

[2] M. S. Baouendi, P. Ebenfelt, and L. P. Rothschild. Rational dependence of smooth and analytic CR mappings on their jets. Math. Ann., 315:205-249, 1999. 
[3] M. S. Baouendi, P. Ebenfelt, and L. P. Rothschild. Dynamics of the Segre varieties of a real submanifold in complex space. J. Algebraic Geom., 12:81-106, 2003.

[4] T. Bloom and I. Graham. On "type" conditions for generic real submanifolds of $\mathbb{C}^{n}$. Invent. Math., 40(3):217-243, 1977.

[5] J. J. Kohn. Boundary behavior of $\bar{\partial}$ on weakly pseudo-convex manifolds of dimension two. J. Differential Geometry, 6:523-542, 1972.

Robert Juhlin

Universität Wien, Fakultät für Mathematik,

Nordbergstrasse 15, A-1090 Wien,

Österreich

E-mail: robert.juhlin@univie.ac.at

Bernhard Lamel

Universität Wien, Fakultät für Mathematik,

Nordbergstrasse 15, A-1090 Wien,

Österreich

E-mail: bernhard.lamel@univie.ac.at

Francine Meylan

University of Fribourg, Department of Mathematics,

CH 1700 Perolles, Fribourg,

Suisse

E-mail:francine.meylan@unifr.ch 